\title{
Recombination and Trapping in Multicrystalline Silicon
}

\author{
Andres Cuevas, Senior Member, IEEE, Matthew Stocks, Daniel Macdonald, Mark Kerr and Chris \\ Samundsett
}

\begin{abstract}
Minority carrier recombination and trapping frequently coexist in multicrystalline silicon (mc-Si), with the latter effect obscuring both transient and steady-state measurements of the photoconductance. In this paper, the injection dependence of the measured lifetime is studied to gain insight into these physical mechanisms. A theoretical model for minority carrier trapping is shown to explain the anomalous dependence of the apparent lifetime with injection level and allow the evaluation of the density of trapping centers. The main causes for volume recombination in mc-Si, impurities and crystallographic defects, are separately investigated by means of cross-contamination and gettering experiments. Metallic impurities produce a dependence of the bulk minority carrier lifetime with injection level that follows the Shockley-Read-Hall recombination theory. Modelling of this dependence gives information on the fundamental electron and hole lifetimes, with the former typically being considerably smaller than the latter, in p-type silicon. Phosphorus gettering is used to remove most of the impurities and reveal the crystallographic limits on the lifetime, which can reach $600 \mu$ s for $1.5 \Omega \mathrm{cm}$ mc-Si. Measurements of the lifetime at very high injection levels show evidence of the Auger recombination mechanism in mc-Si. Finally, the surface recombination velocity of the interface between $\mathrm{mc}-\mathrm{Si}$ and thermally grown $\mathrm{SiO}_{2}$ is measured and found to be as low as $70 \mathrm{~cm} / \mathrm{s}$ for $1.5 \Omega \mathrm{cm}$ material after a forming gas anneal and $40 \mathrm{~cm} / \mathrm{s}$ after an alneal. These high bulk lifetimes and excellent surface passivation prove that mc-Si can have an electronic quality similar to that of single crystalline silicon.
\end{abstract}

Index Terms-Charge carrier processes, photovoltaic cells.

\section{INTRODUCTION}

Despite its extensive and increasing use in the photovoltaic industry, multicrystalline silicon (mc-Si) is, after twenty years of research [1], still a poorly understood material. Inherently, it is a complex semiconductor system where the crystalline lattice is interrupted at the grain boundaries and the volume is frequently populated by foreign atoms, microdefects and dislocations. It is fundamentally nonhomogeneous, with changing properties across the surface of a wafer and within the volume of an ingot. The growth conditions can have a strong effect on its quality and there can be significant differences between ingots grown with the same technique. Furthermore, every one of the several different methods used to grow the material has its own peculiarities.

The characterisation of the electronic properties of mc-Si by means of lifetime testing is complex, owing to its nonhomogeneity and to the co-existence of several different recombination mechanisms. Yet lifetime measurements are the most useful tool available to study the physical limitations of mc-Si and overcome them. It is particularly important to study the possible changes induced by processing [2], a task that is facilitated by the use of simple, contactless measurement techniques. It has recently been realised that the lifetime of mc-Si can be nearly as high as that of single crystal silicon [3,4], that high temperature processing is not necessarily harmful to mc-Si $[5,6]$, that the surfaces can be well passivated [7] and that high efficiency solar cell designs can be implemented in mc-Si wafers [5]. These advancements have led to conversion efficiencies over $18 \%[5,6,8]$ and open-circuit voltages over $650 \mathrm{mV}$ [9]; the $20 \%$ efficiency mark has practically been reached [10]. Nevertheless, besides prime quality material and ultimate performance, research should be directed towards a better understanding of the mid and low quality mc-Si commonly used for mass production of solar cells.

We describe here an study of mc-Si wafers with different dopant densities, contamination levels, crystallographic qualities and surface passivations. The mc-Si material used in this research was produced by directional solidification at Eurosolare SpA [11]. The appropriateness of the quasisteady-state photoconductance method (QSSPC) [12], primarily used for lifetime testing, is discussed first. This method is very convenient to study the variability of the effective minority-carrier lifetime with injection level. Trapping effects, a frequent measurement nuisance, are explained theoretically and used as an additional characterization tool. Controlled cross-contamination of float zone (FZ) single crystal silicon wafers is used to separately study the effect of metal impurities on carrier recombination. Phosphorus gettering is employed to better reveal the limitations imposed by crystallographic defects. Surface passivation by means of thermal oxides and Auger recombination in mc-Si are also investigated. Finally, the improved understanding of multicrystalline silicon gained 
with the previous physical characterization is applied to high performance mc-Si solar cells.

\section{LIFETIME MEASUREMENT TECHNIQUES}

The principles of the quasi-steady-state photoconductance technique (QSSPC) used preferentially in this study for lifetime testing are straightforward [12]. In this technique, the photoconductance of the silicon wafer is measured inductively while it is subjected to a slowly varying illumination, provided by a flash lamp. A steady-state analysis can be used to determine the effective lifetime from the absolute values of the photoconductance and the photo-generation, provided the rate at which the photoconductance adapts to the changing illumination is comparatively fast. Correcting factors $[12,13]$ or quasisteady-state analysis [14] can be used in cases where the previous requirement is not satisfied, thus extending the applicability of the QSSPC method to relatively high lifetimes. This method offers a very convenient way of exploring physical mechanisms in semiconductors over a broad range of carrier injection conditions.

We have found that the transient photoconductance decay (PCD) method frequently overestimates the lifetime of non homogeneous materials like mc-Si. The transient technique has a tendency to emphasize the highest lifetime present in the sample, since the contribution from low-lifetime areas to the photoconductance vanishes quickly and is no longer present in the region of the decay curve typically used to determine the lifetime. On the other hand, a steady-state measurement can be expected to give an area-weighted average of the different lifetimes present in a nonhomogeneous material. For example, a transient PCD measurement of a $1.5 \Omega \mathrm{cm}$ mc-Si wafer gave an effective lifetime of $220 \mu \mathrm{s}$, while the corresponding QSSPC measurement gave $120 \mu$ s [13]. The area sensed by our photoconductance instrument is $4 \mathrm{~cm}^{2}$, approximately. Clearly, steady or quasi-steady state measurements give a more realistic representation of the electronic quality of mcSi.

To investigate possible uncertainties related to the time dependent conditions of the PCD and QSSPC measurements, we have also created true steady-state conditions using two large LED arrays with wavelengths of $660 \mathrm{~nm}$ and $950 \mathrm{~nm}$, respectively. A microprocessor controlled current source provides flexibility and enables, for example, the creation of light pulses with a staircase shape, as shown in Fig.1. The duration of every illumination level can be made appropriately long $(0.5 \mathrm{~ms}$ in Fig.1) to ensure that the photoconductance has reached the steady-state; the overall duration of the light pulse is still short enough to avoid heating the sample. The monotonically decreasing illumination used in the QSSPC technique is, quite obviously, a limiting case of a staircase of true steady-state illumination levels.

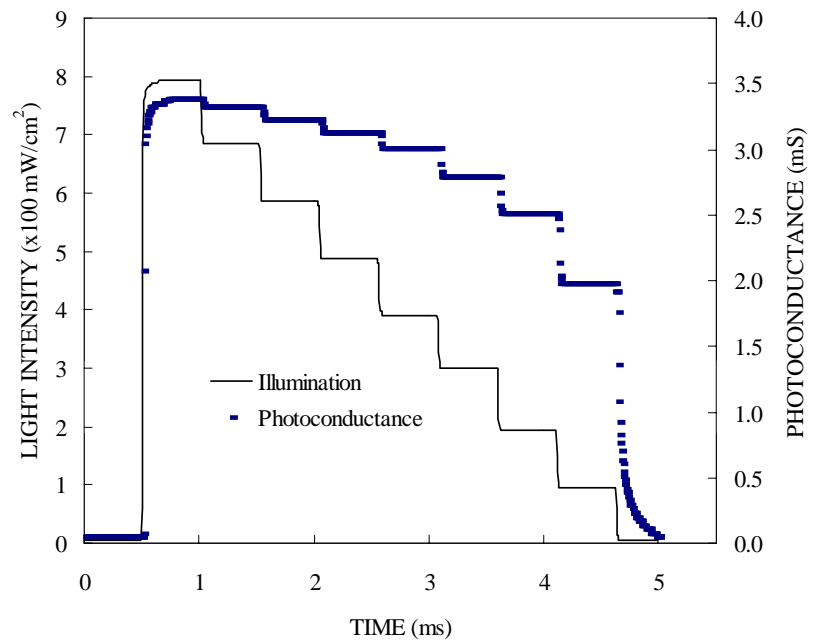

Figure 1. Staircase light pulse $(950 \mathrm{~nm})$ and resulting photoconductance for a mc-Si wafer (sample \#6C, from the central region of an ingot) with pronounced trapping.

\section{CARRIER TRAPPING EFFECTS IN MULTICRYSTALLINE SILICON}

\section{A. Effect of trapping centers on the photoconductance}

Photoconductance measurements of cast mc-Si wafers often show an unusual behaviour at low injection levels. The photoconductance after a light pulse (PCD method) decays very slowly, implying surprisingly high apparent carrier lifetimes, sometimes in the millisecond range. Furthermore, these apparent lifetimes are strongly dependent on the injection conditions, increasing markedly as the carrier density decreases. This strange behaviour is not a measurement artefact resulting from the transient nature of the measurement. In actual fact, the phenomenon is also present in true steady-state measurements of the photoconductance. The $0.5 \mathrm{~ms}$ illumination steps in Fig. 1 are a succession of eight steady-state conditions with light intensities between 1 and 8 standard suns. Note that for 1 sun illumination the photoconductance has reached $60 \%$ of the value corresponding to 8 suns. The ratio between photoconductance and irradiance is indicative of the (apparent) minority carrier lifetime; the latter is much higher (nearly five times) at one sun than at eight suns in this sample. Note also that the incremental change of the photoconductance for equal incremental changes of light intensity becomes smaller as the irradiance increases. Such an strong variability of the photoconductance with the light intensity is a typical manifestation of trapping effects. Of course, the extremely high apparent lifetimes do not represent the true recombination rate of the material. It is, nevertheless, very important to study the effect to gain a better understanding of $\mathrm{mc}-\mathrm{Si}$.

Minority carrier trapping used to plague photoconductance measurements of mono-crystalline germanium and silicon in the early times of crystal growth technology. They 
practically disappeared as the technology matured and produced high quality crystals. It is not surprising that they have resurfaced in $\mathrm{mc}-\mathrm{Si}$, a material that intrinsically contains many crystallographic imperfections. In the '50s and 60 s several authors discussed the physics of carrier trapping and developed mathematical models to describe the effect $[15,16,17,18]$. We have used a simplified form of the model developed by Hornbeck and Haynes [16] to explain the variability of the apparent lifetime with light intensity, adapting it to steady and quasi-steady photoconductance measurements [19].

Although it may sound paradoxical, the main result of minority-carrier trapping is an increase in the concentration of majority carriers. This can be understood as follows. For a given steady-state photo-generation rate, $g_{L}$, in p-type silicon, an excess minority carrier concentration is created so that generation is perfectly balanced by recombination: $\Delta n=g_{L} \tau_{r}$, where $\tau_{r}$ is the recombination lifetime. At the same time, an additional number of electrons $n_{t}$ is trapped. To maintain charge neutrality the concentration of excess majority holes needs to be $\Delta p=\Delta n+n_{t}$. While the trapped electrons are not mobile, the extra holes are, and contribute to the photoconductance:

$$
\Delta \sigma_{L}=q \mu_{n} \Delta n W+q \mu_{p}\left(\Delta n+n_{t}\right) W
$$

where $\mathrm{W}$ is the wafer thickness and $\mu_{n}$ and $\mu_{p}$ are the electron and hole mobilities. The extra photoconductance, given by the term $q \mu_{p} n_{t} W$, can be misleadingly attributed to an abnormally high lifetime.

A theoretical analysis based on the detailed balance of carrier trapping and recombination [16, 19] leads to the following expression for the number of trapped electrons

$$
n_{t}=\frac{N_{t} \Delta n}{\Delta n+N_{t}\left(\tau_{t} / \tau_{g}\right)}
$$

where $\tau_{t}$ is the average time for the capture of electrons by traps and $\tau_{g}$ is the average time for the emission (or regeneration) of electrons from the traps. For typical traps $\tau_{t}<<\tau_{g}$. As eq. (2) shows, $n_{t}$ is dependent on the density of trapping centers, $N_{t}$, and on $\Delta n$, and, since $\Delta n=g_{L} \tau_{r}$, also on the illumination level. The effect of trapping is negligible at sufficiently high illumination levels, when $\Delta n>>n_{t} \approx N_{t}$. It becomes dominant, however, at relatively low illumination levels, when $n_{t}>>\Delta n$.

The model is compared to experimental photoconductance data in Fig. 2. Note that the apparent lifetime changes by almost two orders of magnitude from very low to very high illumination levels. This particular $0.8 \Omega \mathrm{cm}$ wafer came from a region of a cast $\mathrm{mc}-\mathrm{Si}$ ingot between the center and the top, and it had been given a light phosphorus diffusion and oxidation to achieve reliable surface passivation. The model is clearly capable of describing the behaviour of experimental data for a broad range of carrier density levels. The apparent lifetime saturates at high light intensities to a value that is representative of the recombination lifetime, in this case $\tau_{r} \approx 15 \mu$ s. Trapping effects produce a rapid increase of the apparent lifetime at carrier densities in the vicinity of the trap density, in this case $N_{t} \approx 1 \times 10^{15} \mathrm{~cm}^{-3}$. Note that at low injection levels the apparent excess carrier density obtained from a simplistic interpretation of the measured photoconductance does not coincide with the true $\Delta n$. By fitting the model to the experimental data, it is possible to evaluate the density of trapping centres, their energy level $(0.38 \mathrm{eV}$ below the conduction band, approximately) and the ratio between the capture and re-emission characteristic times $\tau_{t} / \tau_{g}=0.004$.



Figure 2. Multicrystalline silicon wafer (\#6B, near the top of the ingot, not gettered) showing strong trapping effects. The apparent lifetime was measured with quasi-steady-state white and infra-red $(1050 \mathrm{~nm})$ illumination, and with steady-state infra-red $(950 \mathrm{~nm}$ LED array) illumination.

Fig. 2 displays data measured with the quasi-steady-state technique using either white light or infra-red illumination. The latter, obtained using a silicon wafer as a filter $(\lambda \approx 1050$ $\mathrm{nm}$ ), produces a uniform photo-generation rate in the $\mathrm{mc}-\mathrm{Si}$ sample and is, therefore, more directly comparable to the theoretical model, which assumes a uniform excess carrier density. Nevertheless, the use of white light does not lead to an important discrepancy in this sample, although it may in other cases. In addition, true steady-state measurements have been made to gain confidence in a particularly strange situation where very long time constants are involved and very drastic changes with illumination conditions can take place. The latter were performed with a $950 \mathrm{~nm}$ LED array using staircase pulses similar to that in Fig.1 (the samples in Figs. 1 and 2 are different, but their trapping behaviour is very similar). The agreement of the steady-sate measurements with the quasi-steady-state ones is excellent.

In our experience, the theoretical model can describe accurately the photoconductance data of mc-Si wafers with 
very different levels of electronic and crystallographic quality. Our experiments [19] indicate that there is a correlation between the densities of trapping centres and dislocations in the material. In addition, we have also found that mobile metallic impurities can produce trapping [20].

Determining the minority carrier lifetime in mc-Si from photoconductance measurements at low injection levels can be misleading in the presence of trapping. Exploring the dependence of the lifetime with injection level is essential to identify the problem. The measured lifetime is only a good reflection of carrier recombination at high injection levels. Nevertheless, the prevailing recombination mechanisms can be different at low and high injection levels and the possibility that the true lifetime in the vicinity of one sun light intensity differs (likely be lower) than at higher irradiances should be kept in mind.

\section{B. Effect of trapping on device voltage}

Given that trapping is an effect that can be observed under steady-state illumination, the question arises as to what extent it might affect the performance of solar cells, in particular their voltage. The voltage created in a solar cell as a consequence of illumination is determined by the product of electron and hole concentrations:

$$
V \cong \frac{k T}{q} \ln \left[\frac{\Delta n\left(N_{A}+\Delta n+n_{t}\right)}{n_{i}^{2}}\right]
$$

where we have used the charge neutrality condition, which, in the presence of traps states that $\Delta p=\Delta n+n_{t}$. In most cases the number of trapped carriers is much lower than the dopant density, $n_{t}<<N_{A}$, and the effect of trapping on device voltage is unnoticeable. It is interesting to note that, in principle, trapping could be beneficial to device voltage, although the effect might only be observable in very lowly doped material. A simple way to interpret the effect of trapping is, according to eq. (3), to consider that it is equivalent to an increased dopant density $N_{A}+n_{t}$.

Note that a prediction of device voltage from measurements of the quasi-steady-state photoconductance needs to take into account the presence of the density of trapped carriers $n_{t}$, given by eq. (2), in eqs. (1) and (3). If $n_{t}$ is ignored, an anomalous dependence of the implicit voltage on light intensity with ideality factors lower than 1 is obtained, as shown in Fig. 3 and ref. [21]. The measurements in Fig.3 correspond to a $1.5 \Omega \mathrm{cm}$ high lifetime mc-Si wafer that produced a solar cell with $\mathrm{V}_{\mathrm{oc}}=644 \mathrm{mV}$, as described in Section VII. After measuring the open-circuit voltage as a function of light intensity the metal contacts were stripped and the photoconductance was measured again. Fig. 3 shows an excellent agreement between the actual voltage and the implied voltage above $550 \mathrm{mV}$, the small difference being attributable to $25 \pm 1{ }^{\circ} \mathrm{C}$ temperature uncertainty. It is interesting to note that, although small, trapping effects are still present at low illumination levels even in this high quality mc-Si wafer.

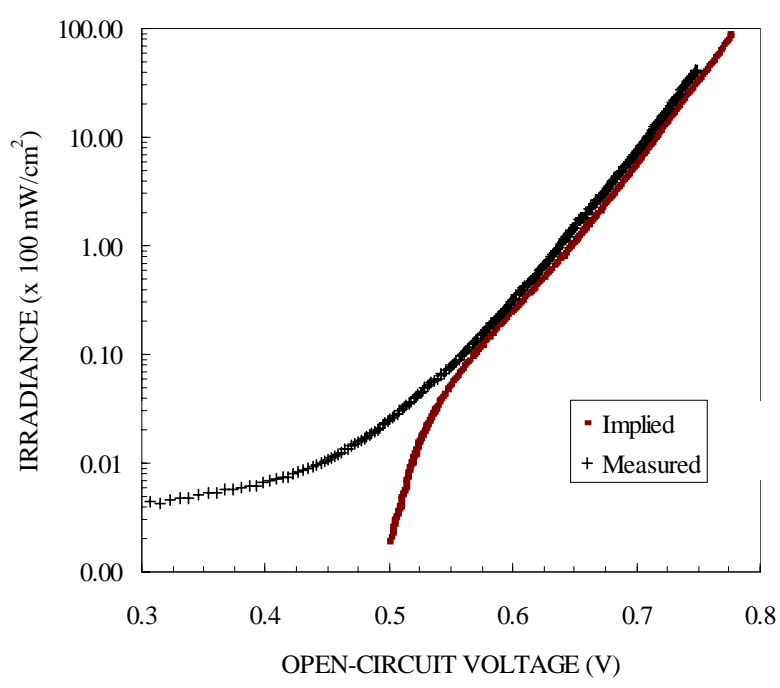

Figure 3. Comparison between the open-circuit voltage implied by a photoconductance measurement and the open-circuit voltage actually measured for a high performance multicrystalline silicon solar cell.

\section{VOLUME RECOMBINATION IN MULTICRYSTALLINE SILICON}

Besides being crystallographically imperfect, cast mc-Si ingots usually contain a high concentration of metallic impurities. Typically, both the defect density and the concentration of impurities vary along the vertical and horizontal dimensions of the ingot and also from ingot to ingot, making it difficult to separate their respective effect on volume recombination. In this section we present two techniques that are useful to investigate these two causes of bulk lifetime degradation. The first is based on the crosscontamination of hyper-pure, defect-free wafers. The second consists of gettering the metallic impurities to reveal the role of crystallographic defects more clearly.

\section{A. Recombination due to metallic impurities}

The concentration of metallic impurities in mc-Si wafers can be measured using a variety of techniques, although the measurements can be quite difficult, particularly if the density of contaminants is low. Lifetime testing offers an alternative that can have a greater sensitivity. We have developed a simple method based on the crosscontamination that occurs between mc-Si wafers and high purity mono-crystalline $\mathrm{Si}$ wafers placed in close proximity during a high temperature process. A proportion of the mobile impurities present in the multicrystalline wafers effuses out of them and is absorbed by adjacent monocrystalline wafers. The lifetime of the control wafers decreases monotonically with increasing concentration of mobile impurities in the corresponding $\mathrm{mc}-\mathrm{Si}$ wafer, 


\begin{tabular}{|c|c|c|c|c|c|c|c|}
\hline \multirow[t]{2}{*}{ Sample } & \multirow[t]{2}{*}{ Ingot region } & \multirow{2}{*}{$\begin{array}{c}\text { mc-Si } \tau_{\text {eff }} \\
\text { pre-getter } \\
(\mu \mathrm{s})\end{array}$} & \multicolumn{3}{|c|}{ FZ-Si control wafers } & \multirow{2}{*}{$\begin{array}{l}\text { Dislocation } \\
\text { density } \\
\left(\mathrm{cm}^{-2}\right)\end{array}$} & \multirow{2}{*}{$\begin{array}{c}\text { Mc-Si } \tau_{e f f} \\
\text { after getter } \\
\quad(\mu \mathrm{s})\end{array}$} \\
\hline & & & $\begin{array}{r}\tau_{\text {eff }} \\
(\mu \mathrm{s})\end{array}$ & $\begin{array}{l}\tau_{n o} \\
(\mu \mathrm{s})\end{array}$ & $\begin{array}{l}\tau_{p o} \\
(\mu \mathrm{s})\end{array}$ & & \\
\hline $6 \mathrm{~A}$ & top & 1 & 130 & 35 & 110 & $6 \times 10^{6}$ & 1 \\
\hline $6 B$ & near top & 15 & 180 & 45 & 140 & $6 \times 10^{5}$ & 40 \\
\hline $6 \mathrm{C}$ & center & 35 & 800 & 210 & 660 & $5 \times 10^{5}$ & $40-50$ \\
\hline $6 \mathrm{D}$ & bottom & 2 & 300 & 84 & 260 & $1 \times 10^{4}$ & 20 \\
\hline
\end{tabular}

Table I. Effective lifetime $\tau_{\text {eff }}$ before and after phosphorus gettering, and dislocation density of wafers from different regions of a $0.8 \Omega \mathrm{cm}$ mcSi ingot. Only the highest values of $\tau_{\text {eff }}$ are given, although it may vary with injection level. The highest $\tau_{\text {eff }}$ of the corresponding crosscontaminated $1000 \Omega \mathrm{cm}$ float zone $\mathrm{Si}$ wafers and their fundamental electron, $\tau_{n o}$, and hole, $\tau_{p o}$, lifetimes are also given.

allowing "clean" and "contaminated" mc-Si material to be discriminated [22].

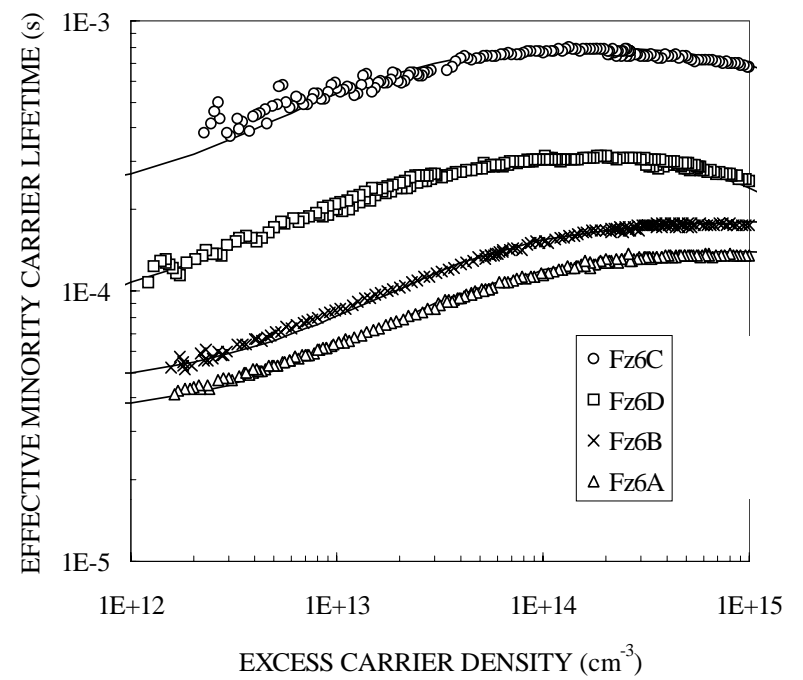

Figure 4. Lifetime as a function of the excess carrier density for cross-contaminated $1000 \Omega \mathrm{cm}$ single crystal silicon wafers. The source of contamination for samples Fz6C, Fz6D, Fz6B and Fz6A are the center, bottom, near top and top regions of a mc-Si ingot, respectively. The continuous lines represent the Shockley-ReadHall theory.

Several cross-contamination experiments consisting of a light $\mathrm{POCl}_{3}$ diffusion at $840^{\circ} \mathrm{C}$ for 25 minutes followed by thin oxide layer growth at $900^{\circ} \mathrm{C}$ for 30 minutes, were performed on wafers from various regions of a cast $\mathrm{mc}-\mathrm{Si}$ ingot. Considering that the effective lifetime of a clean $1000 \Omega \mathrm{cm} F Z$ wafer processed in an identical way is typically 1-2 $\mathrm{ms}$, the results in Table I indicate that the concentration of mobile impurities is low in the central region (control wafer lifetime $800 \mu$ s), while it is high at the bottom of the ingot (most likely due to contamination from the crucible), and also at the top (caused by segregation of metallic impurities during ingot growth) [23].
Subsequent SIMS measurements detected the presence of chromium and iron in the mc-Si wafers. We tried to verify the presence of iron in cross-contaminated FZ-Si wafers (a lower resistivity, $1 \Omega \mathrm{cm}$, was used for this experiment) by means of the thermal dissociation of iron-boron pairs [24], but the expected degradation of the lifetime after a $210{ }^{\circ} \mathrm{C}$ anneal could not be observed, suggesting that the concentration of iron is probably low. The presence of chromium and other metals is still possible and could explain the measured lifetimes [25].

In an elegant way, the cross-contamination technique transfers metallic impurities to a crystallographically perfect medium where their effect on carrier recombination can be studied without the combined influence of grain boundaries, dislocations and other defects present in the mc-Si wafers. In fact, trapping effects make it impossible to explore the low carrier density range in many mc-Si wafers. It is possible to investigate fundamental properties of carrier recombination such as the capture cross-sections of electrons and holes by analyzing the dependence of the minority carrier lifetime on injection level. Fig. 4 shows the effective lifetime, $\tau_{\text {eff }}$, of cross-contaminated $1000 \Omega \mathrm{cm} \mathrm{FZ}$ wafers measured with QSSPC and PCD techniques. The surfaces of the wafers were passivated with a light phosphorus diffusion and oxidation, allowing to consider the measured lifetime to be an accurate representation of bulk recombination. The increase of the lifetime with injection level apparent in Fig. 4 suggests that ShockleyRead-Hall $[17,18]$ is the dominant recombination mechanism. This theoretical model predicts for p-type material and recombination centers located in the middle of the energy gap the following expression for the bulk minority carrier lifetime:

$$
\tau_{(S R H)}=\tau_{n o}+\tau_{p o} \frac{\Delta n}{\Delta n+N_{A}}
$$

The experimental lifetime data for the cross-contaminated wafers in Fig. 4 are in excellent agreement with the model expressed by eq. (4). By fitting the model to the measured data, it is possible to determine the fundamental electron, $\tau_{n o}$, and hole, $\tau_{p o}$, lifetimes. The resulting values, given in 
Table I, indicate that the ratio $\tau_{n o} / \tau_{p o}$ is essentially constant, equal to 0.32 , for the four samples. This implies a common type of recombination center; its concentration varies for the different control wafers and, therefore, for the different regions of the mc-Si ingot.

\section{B. Phosphorus gettering. Crystallographic limits to recombination}

To complete the characterization of the mc-Si wafers in the previous section, they were subjected to a $\mathrm{POCl}_{3}$ gettering treatment followed by silicon etch and an additional light diffusion to passivate the surfaces. The FZ controls, which were also gettered, recovered the high lifetimes typical of the uncontaminated state. The gettered mc-Si wafers did not produce further cross-contamination, indicating that the $3 \mathrm{~h}$, $900{ }^{\circ} \mathrm{C}$ gettering treatment was sufficient to extract a majority of the mobile metal atoms. The final lifetimes of the gettered mc-Si wafers are a clearer measure of their crystallographic quality than the lifetimes before gettering. This quality proved poorest at the top of the ingots, with lifetimes of the order of $1 \mu \mathrm{s}$, compared to $40-50 \mu \mathrm{s}$ in the central regions (see Table I).

As expected, most of the mc-Si wafers that had been identified as containing a high density of mobile impurities by the previous cross-contamination experiment improved markedly with phosphorus gettering, indicating that those impurities were the main factor limiting their initial lifetime. Nevertheless, some wafers that also effused many impurities did not improve with gettering. Typically, this happened to wafers from the upper region of the ingot, which had a very high density $\left(>10^{6} \mathrm{~cm}^{-2}\right)$ of dislocations. A correlation between lack of response to gettering and high dislocation density has also been observed by other authors [26]. Recent research has shown that metal impurities, including iron, chromium and nickel, precipitate at dislocation clusters in $\mathrm{mc}-\mathrm{Si}$ and produce high carrier recombination [27]; it seems that those impurity agglomerates are not easily gettered. In a few cases we also found a lack of response to gettering in samples from the lower region of the ingot [28], despite the lower dislocation density in Table I. We attribute this to a likely horizontal variability of the dislocation density, which was measured only on a $12 \mathrm{~cm}^{2}$ sample of every vertical region. It is clear that a cross-contamination experiment is not enough to predict the response to gettering and needs to be complemented with a measurement of the dislocation density.

\section{High effective minority-carrier lifetimes in $\mathrm{mc}-\mathrm{Si}$}

Using phosphorus gettering and good quality $1.5 \Omega \mathrm{cm}$ mcSi wafers, we have measured effective lifetimes higher than $200 \mu \mathrm{s}$ [3]. Note that the measured lifetime may include contributions from both the bulk of the material and the surfaces, although the latter was minimized by passivating them with a light phosphorus diffusion and oxidation. The quality of this mc-Si material compares favourably to monocrystalline $\mathrm{CZ}$ silicon of the same resistivity.
In a similar way to single crystal silicon, we have observed in mc-Si a strong dependence of the lifetime on dopant density. P-type $0.4 \Omega \mathrm{cm}$ wafers have showed lifetimes up to $90 \mu$ s after phosphorus gettering, and lifetimes up to $15 \mu \mathrm{s}$ have been measured in $0.2 \Omega \mathrm{cm} \mathrm{mc}-\mathrm{Si}$, which is remarkable for material doped with $10^{17} \mathrm{~cm}^{-3}$ boron atoms. Table II summarizes the highest measured values of $\tau_{\text {eff }}$, although it should be kept in mind that $\tau_{\text {eff }}$ may vary with injection level. It is important to note that the photoconductance testing apparatus measures an area that includes several grain boundaries. The high lifetimes that we have observed indicate that these grain boundaries are quite inactive electronically.

\begin{tabular}{|l|l|l|l|}
\hline Resistivity & $0.2 \Omega \mathrm{cm}$ & $0.4 \Omega \mathrm{cm}$ & $1.5 \Omega \mathrm{cm}$ \\
\hline $\begin{array}{l}\text { Pre-gettered } \\
\tau_{\text {eff }}(\mu \mathrm{s})\end{array}$ & 10 & 40 & 70 \\
\hline $\begin{array}{l}\text { Gettered } \\
\tau_{\text {eff }}(\mu \mathrm{s})\end{array}$ & 15 & 90 & 260 \\
\hline
\end{tabular}

Table II: Best effective minority carrier lifetimes measured in mc$\mathrm{Si}$ material of different resistivities, showing the effect of phosphorus gettering.

\section{SURFACE RECOMBINATION VELOCITY OF MULTICRYSTALLINE SILICON}

The successful improvement of the minority carrier lifetime in the bulk of mc-Si wafers brings about recombination at the surfaces as an extremely relevant performance-limiting mechanism. The present trend towards very thin silicon solar cells accentuates the importance of the surfaces. Although well known for single crystal silicon, the surface recombination velocity of the silicon/silicon dioxide interface of $\mathrm{mc}-\mathrm{Si}$ had not been characterized before. The experiments that we have used to separate bulk and surface recombination are relatively involved since several identical mc-Si wafers of different thicknesses are needed. The surface recombination velocity $S$ can then be obtained by plotting the measured effective lifetime as a function of the thickness, using the following expression:

$$
\frac{1}{\tau_{\text {eff }}}=\frac{1}{\tau_{\text {bulk }}}+2 \frac{S}{W}
$$

Fig. 5 shows the surface recombination velocity of high quality, phosphorus-gettered $1.5 \Omega \mathrm{cm} \mathrm{mc-Si} \mathrm{wafers} \mathrm{that}$ were subjected to a thermal oxidation in steam at $900{ }^{\circ} \mathrm{C}$ for 2 hours followed by an argon anneal at the same temperature for 20 minutes and a FGA at $400{ }^{\circ} \mathrm{C}$. Two wafer thicknesses, $120 \mu \mathrm{m}$ and $200 \mu \mathrm{m}$ were used to extract the surface recombination velocity, with a third, $430 \mu \mathrm{m}$ thick, wafer providing additional verification of the bulk lifetime. The quality of the surface passivation is remarkably good, with $S \approx 70-120 \mathrm{~cm} / \mathrm{s}$ in the carrier density range $10^{15} \mathrm{~cm}^{-3}$ to $5 \times 10^{16} \mathrm{~cm}^{-3}$. Measurements at lower carrier densities were impeded by trapping effects in these wafers. In separate experiments using a different set of 
samples we also observed surface recombination velocities in the $75-125 \mathrm{~cm} / \mathrm{s}$ range for steam oxide, while oxidations in dry oxygen with TCA at $1000{ }^{\circ} \mathrm{C}$ were found to give an slightly poorer passivation $S \approx 175-300 \mathrm{~cm} / \mathrm{s}$ [7]. Bearing in mind that mc-Si is intrinsically diverse and may behave differently, the present experiments indicate that it can be well passivated using similar techniques to single crystal silicon, and that similarly low values of $S$ can be achieved.



Figure 5. Surface recombination velocity of oxidized $1.5 \Omega \mathrm{cm}$ mcSi wafers. The two sets of data correspond to a forming gas anneal and an aluminium anneal, respectively.

We investigated the effect of an aluminium anneal, or alneal, on the surface recombination velocity. The same samples were used in this experiment, where a $0.1 \mu \mathrm{m}$ layer of pure aluminium was deposited by thermal evaporation on both sides of the wafers. After a 30 minutes, $400{ }^{\circ} \mathrm{C}$ anneal in forming gas the aluminium was etched, leaving the silicon dioxide intact. The resulting surface recombination velocity has also been plotted in Fig.5. It shows a moderate injection-dependence, with a minimum of $40 \mathrm{~cm} / \mathrm{s}$ at a carrier density of $10^{15} \mathrm{~cm}^{-3}$, approximately ten times lower than the dopant density of these wafers. The significant improvement in surface passivation obtained with an alneal in $\mathrm{mc}-\mathrm{Si}$ is, again, similar to that reported for single crystal silicon. The actual passivation achieved in some high efficiency solar cells may be still better at the rear surface, where the aluminium layer is usually kept as an optical reflector. The situation may be different at the front surface, where the passivating effect of the alneal is likely to be unstable.

\section{AUGER RECOMBINATION IN MULticRYSTALline SILICON}

The variable thickness experiment described in the previous section also permits a direct evaluation of the bulk minority carrier lifetime. The results corresponding to the same phosphorus-gettered $1.5 \Omega \mathrm{cm}$ mc-Si wafers used in Section $\mathrm{V}$ are shown in Fig. 6. The bulk minority carrier lifetime in low injection for the case of forming gas annealed wafers is in good agreement with our previous measurements [7] on similar material, with $\tau_{\text {bulk }} \approx 100-300 \mu \mathrm{s}$. A significant improvement occurs after the alneal, with a maximum lifetime of approximately $\tau_{\text {bulk }} \approx 600 \mu$ s at an excess carrier density of $10^{16} \mathrm{~cm}^{-3}$. At lower carrier densities, the lifetime decreases to about $200 \mu$ s following a Shockley-Read-Hall dependence.

At higher injection levels, that is, excess carrier density greater than $10^{16} \mathrm{~cm}^{-3}$, the lifetime drops according to the quadratic dependence on carrier density characteristic of the Auger recombination mechanism. The measured data is in excellent agreement with the Auger coefficient $\mathrm{C}_{\mathrm{A}} \approx 1.66 \times 10^{-30} \mathrm{~cm}^{-6} \mathrm{~s}^{-1}$ measured by other researchers in single crystal silicon [29]. Although it is reasonable to expect that the Auger mechanism, being a fundamental characteristic of the material, should not be related to its crystallographic properties, it is reassuring to have an experimental confirmation of its occurrence in $\mathrm{mc}-\mathrm{Si}$.

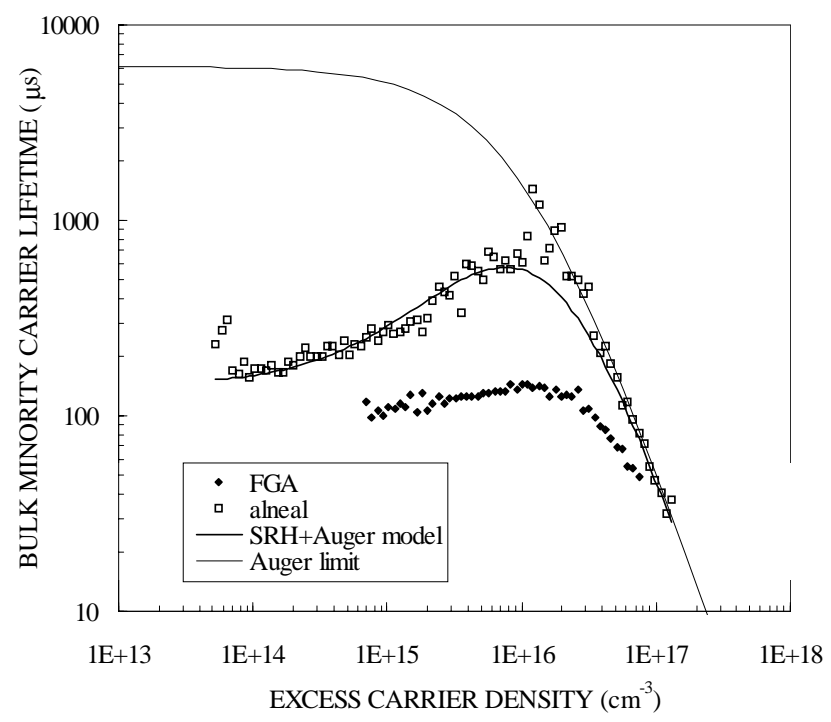

Figure 6. Bulk minority carrier lifetime of $1.5 \Omega \mathrm{cm}$ mc-Si wafers. The two sets of data correspond to a forming gas anneal and an aluminium anneal, respectively. The continuous line for the alneal case represents the Shocley-Read-Hall and Auger theoretical modelling of bulk recombination.

\section{COMPARISON WITH SOLAR CELL VOLTAGES}

The electrical characteristics of the best solar cells made with phosphorus-gettered $\mathrm{mc}-\mathrm{Si}$ of three different resistivities are given in Table III. It is instructive to work out the effective lifetime implicit in the final device voltage [30]. From the measured $V_{o c}$, the excess carrier density can be evaluated, while $J_{s c} / q$ can be used as a good approximation (a lower bound) to the total photo-generation 
in the wafer. The implicit lifetimes for the three cells in Table III are, approximately, $6 \mu \mathrm{s}, 30 \mu \mathrm{s}$ and $75 \mu_{\mathrm{s}}$ for the $0.2 \Omega \mathrm{cm}, 0.4 \Omega \mathrm{cm}$ and $1.5 \Omega \mathrm{cm}$ substrates, respectively. Note that these are effective lifetimes that include bulk, surface and diffused region recombination; an additional difference with the mc-Si samples discussed in previous sections is that the finished cells have metal contacts. It is understandable that the implied device lifetimes are lower; still, they are remarkable, considering that several high temperature steps are used for the fabrication of the devices.

The similarity of the short-circuit current densities given in Table III can be misleading if the different antireflection coatings and surface faceting of the cells are not considered: tubs and encapsulation for the $0.2 \Omega \mathrm{cm}$ cell, double layer antireflection coating on a flat surface for the $0.4 \Omega \mathrm{cm}$ device and a crude single layer of $\mathrm{SiO}_{2}$ on flat surface for the $1.5 \Omega \mathrm{cm}$ solar cell. The rear surface is also different, with a $100 \%$ metal contact, oxide passivation with $5 \%$ local contact, and oxide passivation with local boron contact for the $0.2 \Omega \mathrm{cm}, \quad 0.4 \Omega \mathrm{cm}$ and $1.5 \Omega \mathrm{cm}$ solar cells, respectively.

Interestingly, the voltage of the $1.5 \Omega \mathrm{cm}$ cell increased 11 $\mathrm{mV}$ with an alneal of the front surface. This is at least partially due to the improved passivation of the oxide that surrounds the $2 \times 2 \mathrm{~cm}^{2}$ active area of the cell (the aluminium was only etched-off the active area). This gives additional proof of the beneficial effect of an alneal on cell performance. This treatment was not performed on the other two cells in Table III.

\begin{tabular}{|l|l|l|l|l|l|}
\hline $\begin{array}{l}\text { Resistivity } \\
(\Omega \mathrm{cm})\end{array}$ & $\begin{array}{l}\mathrm{J}_{\mathrm{sc}} \\
\left(\mathrm{mA} / \mathrm{cm}^{2}\right)\end{array}$ & $\begin{array}{l}\mathrm{V}_{\mathrm{oc}} \\
(\mathrm{mV})\end{array}$ & $\begin{array}{l}\tau_{\text {implic }} \\
(\mu \mathrm{s})\end{array}$ & FF & $\begin{array}{l}\text { Eff. } \\
(\%)\end{array}$ \\
\hline 0.2 & 34.9 & 657 & 6 & 0.81 & 18.6 \\
\hline 0.4 & 34.8 & 654 & 28 & 0.8 & 18.2 \\
\hline 1.5 & 34.2 & 644 & 75 & 0.79 & 17.5 \\
\hline
\end{tabular}

Table III: Best $4 \mathrm{~cm}^{2}$ multicrystalline Si solar cells made at the $A N U$. The cells have different optical properties and internal structure.

\section{CONCLUSIONS}

Multiple recombination mechanisms usually coexist in multicrystalline silicon, and their characterisation is frequently hindered by carrier-trapping effects. The measurement and analysis of the injection dependence of the effective minority-carrier lifetime provides insight into these mechanisms.

Trapping is manifested by an abnormally high apparent lifetime at low illuminations that decreases drastically with increasing injection level. We have found that the experimental data are adequately explained by a theoretical model developed by Hornbeck and Haynes in 1954, which permits the determination of the density of trapping centers. The origin of these traps is still uncertain, with preliminary evidence indicating that they are related to the crystallographic quality of the material, including grain size, dislocation density and micro-defects, and also to metal impurities. The study of these trapping effects may become a useful tool to diagnose the quality of multicrystalline silicon.

Because of trapping, a photoconductance-based measurement of the recombination parameters in mc-Si at the low injection levels pertinent to solar cell operation is not possible in many cases. By transplanting the impurities present in the mc-Si to single crystal wafers it is possible to see that they make the effective lifetime increase with carrier density at low and intermediate injection levels. This behaviour is typical of Shockley-Read-Hall recombination, and the strong variability of $\tau_{\text {bulk }}$ observed in heavily contaminated samples indicates that there is an asymmetry between the electron and hole fundamental lifetimes in those metal-related recombination centers. A similar dependence can be expected to occur in the mc-Si wafers that were the source of contamination; this means that an extrapolation of the lifetime measured at high light intensity to one-sun conditions may be optimistic in some cases. Purification at the wafer level can be satisfactorily achieved by means of phosphorus gettering. This minimizes, but does not completely exclude, the impact of impurities on volume recombination. Additional defects remain after gettering in common mc-Si and limit its lifetime to values well below those found in mono-crystalline material.

The measurement of surface recombination velocities is also compromised by trapping effects. In the range of carrier densities where clean measurements have been possible, we have found that the SRV of thermally oxidized mc-Si is as low as for single crystalline silicon of the same resistivity, despite the multiple crystallographic orientation of mc-Si. We have also observed a significant improvement of the passivation quality of thermal oxides with an alneal, and measured a minimum surface recombination velocity of $40 \mathrm{~cm} / \mathrm{s}$.

Some mc-Si material has shown an impressive electronic quality, with minority carrier lifetimes as high as $600 \mu \mathrm{s}$, implying a minority carrier diffusion length in excess of 1 $\mathrm{mm}$. This quality supports the feasibility of mc-Si cells with efficiency greater than $20 \%$. Nevertheless, size is important, and high efficiency laboratory devices are usually very small in comparison to commercial solar cells. Ingot growth techniques need to be improved to produce mc-Si with a sufficiently uniform quality to achieve high performance in large area solar cells. The techniques used in this work to understand the fundamental electronic mechanisms in mc$\mathrm{Si}$ better could be useful in this endeavour.

\section{ACKNOWLEDGMENT}

The multicrystalline silicon wafers used in this research have been kindly provided by Dr F. Ferrazza of Eurosolare 
SpA. Christopher Jones designed and built the LED light source. Dr R. Sinton contributed with useful discussions and suggestions. Funding was provided by the Australian Research Council.

\section{REFERENCES}

[1] J. Lindmayer, "Semicrystalline silicon solar cells", Proc. 12 $2^{\text {th }}$ IEEE Photovoltaic Specialists Conf., p. 82, 1976.

[2] M. Stocks, A. Cuevas and A. Blakers, "Minority Carrier Lifetimes of Multicrystalline Silicon During Solar Cell Processing", In Proc. $14^{\text {th }}$ European Photovoltaic Solar Energy Conf., Barcelona, July 1997, H.S. Stephens, Falmerston, UK, 1997, pp. 770-773.

[3] A. Cuevas, M.J.Stocks, S. Armand, M.Stuckings, A.W. Blakers and F. Ferrazza, "High minority carrier lifetimes in multicrystalline silicon", Appl. Phys. Lett., vol 70 (8), pp. 1017-1019, February 1997.

[4] H. Nagel, J, Schmidt, A. Aberle and R. Hezel, "Exceptionally high bulk minority-carrier lifetimes in block-cast multicrystalline silicon", Proc. $14^{\text {th }}$ European Conf. on Photovoltaic Solar Energy, Barcelona, pp. 762-765, 1997.

[5] M. Stocks, A. Blakers and A. Cuevas, "Multicrystalline Silicon Solar Cells with Low Rear Surface Recombination", Proc. $26^{\text {th }}$ IEEE Photovoltaic Specialists Conf., Anaheim, Sept. 1997, pp. 67-70.

[6] J. Zhao, A. Wang and M. Green, "High efficiency multicrystalline solar cells using standard float-zoned processing", Progress in Photovoltaics, vol 5, pp. 169-174, 1997.

[7] M. Stocks and A. Cuevas, "Surface recombination velocity of thermally oxidised multicrystalline silicon", Proc. Second World Conf. on Photovoltaic Energy Conversion, Vienna, July, 1998, to be published.

[8] A. Rohatgi, S. Narashima, S. Kamra, P. Doshi, C. Khattak, K. Emery and H. Field, "Record high $18.6 \%$ efficient solar cell on HEM multicrystalline material", Proc. $25^{\text {th }}$ IEEE Photovoltaic Specialists Conf., Washington, May 1996, p. 1554.

[9] M. Stocks, A. Blakers and A. Cuevas, "Record Open Circuit Voltage Multicrystalline Silicon Solar Cells", IEEE Trans. on Electron Devices, accepted for publication, 1998.

[10] J. Zhao, A. Wang, and M. Green, " $19.8 \%$ efficient "honeycomb" textured multicrystalline silicon solar cells", Appl. Phys. Lett., pp. 1991-1993, Oct. 1998

[11] F. Ferrazza, L. Modica, D. Margadona and M. Rustioni, "Basic requirements for solar grade feedstock", Proc. $12^{\text {th }}$ European Conf. on Photovoltaic Solar Energy, Amsterdam, 1994, pp. 1007-1008.

[12] R. A. Sinton and A. Cuevas, "Contactless determination of current-voltage characteristics and minority-carrier lifetimes in semiconductors from quasi-steady-state photoconductance data" Appl. Phys.Lett., Vol. 69(17), pp. 2510-2512, October 1996.

[13] M. Stocks, "High efficiency multicrystalline silicon solar cells", $\mathrm{PhD}$ thesis, Australian National University, 1998.

[14] H. Nagel, C. Berge and A. Aberle, "Generalized analysis of transient and quasi-steady-state photoconductance data for carrier lifetime measurements", private communication, unpublished

[15] H.Y. Fan, "Effect of traps on carrier injection in semiconductors", Phys. Rev., vol. 92, pp. 1424-1428, 1953.

[16] J.A. Hornbeck and J.R. Haynes, "Trapping of minority carriers in silicon", Phys. Rev., vol. 97, pp. 311-321, 1955.

[17] R. A. Smith, Semiconductors, Cambridge Press, 1959.

[18] J.S. Blakemore, "Semiconductor Statistics", Pergamon Press, 1962.

[19] D. Macdonald and A. Cuevas, "Minority-carrier trapping in multicrystalline silicon", Appl. Phys. Lett., vol.74, pp. 1710-1712 (1999).

[20] D. Macdonald and A. Cuevas, "Boron-related minority carrier traps in p-type silicon", submitted to Appl. Phys. Lett.

[21] R. Sinton, A. Cuevas and M. Stuckings, "Quasi-steady-state photoconductance, a new method for solar cell material and device characterization", Proc. 25th IEEE Photovoltaic Specialists Conf., Washington, pp. 547-550 (1996).

[22] D. Macdonald and A. Cuevas, "Cross-contamination as a novel technique for studying impurities in multicrystalline silicon", Proc. Second World Conf. on Photovoltaic Energy Conversion, Vienna, July 1998, pp. 2418-2422 (1998).
[23] F. Ferrazza, Polycrystalline Semiconductors IV - Physics, Chemistry and Technology, Trans. Tech. Publ., Edited by Pizzini, S. Strunk, H. P., Werner, J. H., (1995).

[24] G. Zoth and W. Bergholz, " A fast, preparation-free method to detect iron in silicon, J.Appl. Phys., vol. 67, pp.6764-6771, 1990.

[25] M.L. Polignano, F. Cazzaniga, A. Sabbadini, F. Zanderigo, and F. Priolo, "Metal contamination monitoring and gettering", Materials Science in Semiconductor Processing, vol. 1, pp. 119-130 (1998).

[26] S.A. McHugo, J. Bailey, H. Hieslmair, and E. R. Weber, "Efficiency-limiting defects in polycrystalline silicon", Proc. First World Conf. on Photovoltaic Energy Conversion, Hawaii, Dec. 1994 pp. 1607-1610.

[27] S.A. McHugo, A.C. Thompson, I. Perichaud and S. Martinuzzi, "Direct correlation of transition metal impurities and minority carrier recombination in multicrystalline silicon", Appl. Phys. Lett., vol. 72, pp. 3482-3484, 1998.

[28] D. Macdonald, A. Cuevas and F. Ferrazza, "Response to phosphorus gettering of different regions of cast multicrystalline silicon ingots", Solid-State Electronics, vol. 43, pp. 575-581 (1999).

[29] R.A. Sinton and R.M. Swanson, "Recombination in highly injected silicon", IEEE Trans. Electron Devices, vol. ED-34, pp. 1380-1389, 1987.

[30] A. Cuevas and R. A. Sinton, "Prediction of the open circuit voltage of solar cells from the steady-state photoconductance", Progress in Photovoltaics, Vol. 5, pp. 79-90, March 1997. "Semicrystalline silicon solar cells", Proc. $12^{\text {th }}$ IEEE Photovoltaic Specialists Conf., p. 82, 1976. 